\title{
Study of LED Electronic Writing Screen based on Single Chip Dengjin $\mathrm{Wu}^{1}$ \\ ${ }^{1}$ North China Electric Power University, Beijing, 10206 \\ hunter2011@foxmail.com
}

Keywords: STC89C52; LED Color Dot Matrix; Infrared Phototransistor Light Pen

\begin{abstract}
LED display design hardware consists of STC89C52 smallest single-chip system, $32 \times$ 32 dot matrix color display arrays, light pen, buttons, LCD display and other components. Color dot matrix red LED is always working in dimly lit scan status, lit STC89C52 microcontroller use homemade light pen infrared phototransistor to detect light strokes and position the red LED lights, calculate from the ranks of coordinate light pen position, and according to key settings different operating mode to control LED display, enable lit, scratch, reverse display, the screen is cleared, strokes drag, rotate display. Display according to ambient light intensity automatically adjusts the display brightness of the display, within a set time when the light pen is not touching the display or button is not pressed close all display and the system enters hibernation, reduce power consumption, when a key press the system resumes.
\end{abstract}

\section{Introduction}

LED closely watched since birth, LED is called the fourth generation light source or green light, energy saving, environmental protection, long life, small size and other characteristics, widely used in various directions, display, decoration, backlight, general urban landscape lighting and other fields. One especially in the 21st century, LED display as the leading flat panel display products will undoubtedly have a greater development, and there may be twenty-first century representation of mainstream flat panel display products. Dot matrix LED display module using the dot or pixel unit composed of the light-emitting diode display screen variable area with high reliability, long life, environmental adaptability, high cost performance, low cost, has been as many outdoor display media and electronic tools work displayed, widely used in railway stations, hotels, finance, securities, telecommunications and sports advertising or transportation and other industries.

LED display current design has a variety of methods can be achieved, but the market can only be done using the electronic display simple display effect. Achieve more functionality on the PC to be in reality. This work is based on single-chip design STC89C52 the corresponding light pen detection module circuit and led the writing circuit diagram display modules, transistors induction led from the faint light emitted, thereby resistance to change, resulting in changes in high and low, the level of using SCM level analog signal into a digital signal, by program control, the signal is passed to each module, and then converted to an analog signal, through change in the potential of the corresponding led is lighted, and lcd led writing display screen the coordinates of the corresponding led is lit on the display, and control LED display depending on the mode button set to achieve lighting, zoned light, reverse display, the screen is cleared, strokes drag, rotate display. Make led display more controllable.

\section{System Design}

The Overall System Design. According to the photoelectric effect, PN junction in the excitation light can change its resistivity, while the LED is a PN junction, so the possibility of using PN junction characteristics LED dot matrix light-sensitive screen to complete it? After a simple experiment to explore, we find LED itself has good photosensitivity, this way, the screen can take full advantage of the photosensitive characteristics of the photosensitive LED to achieve. In this paper, the use of a $8 \times 8$ dot matrix screen, combined with a STC89C52 MCU having a photosensitive function of lattice screen, shown in Figure 1. 


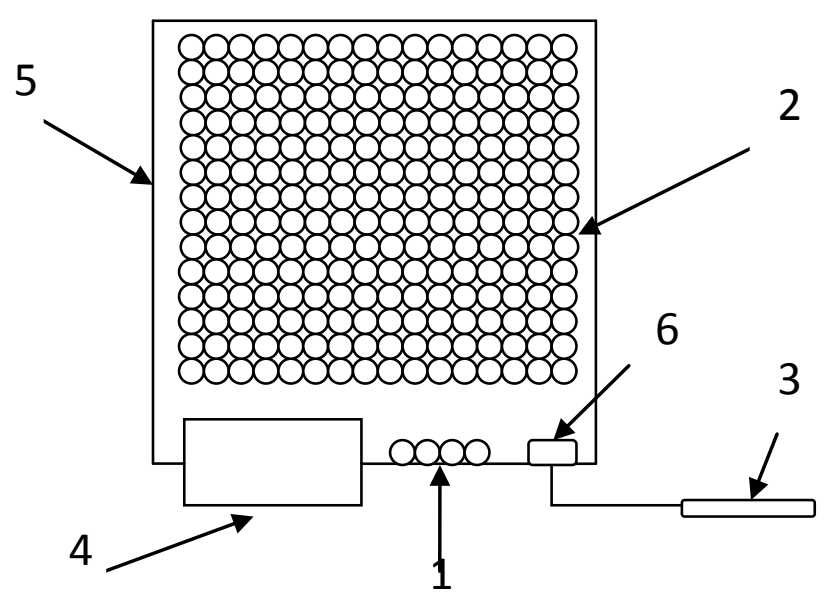

Fig. 1 LED iting display overall view

Where: 1: Key Module 2: led dot matrix screen writing 3: light pen detection module 4: LCD Display Module 5: led writing display enclosure 6: Wiring mouth

System consists of a microprocessor STC89C52, $32 \times 32$-color LED dot matrix display, a light pen and the detection circuit, the external light intensity detecting circuit, a key input circuit, the liquid crystal display module composed of several parts.

There STC89C52 the microcontroller chip chip RAM 1KB, it is possible to save the four-screen display information to meet the requirements, the high cost microcontroller. System diagram shown in Figure 2.

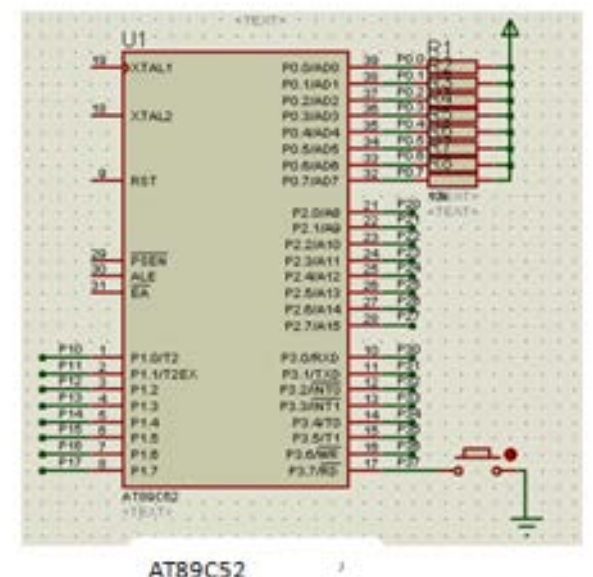

AT89C52
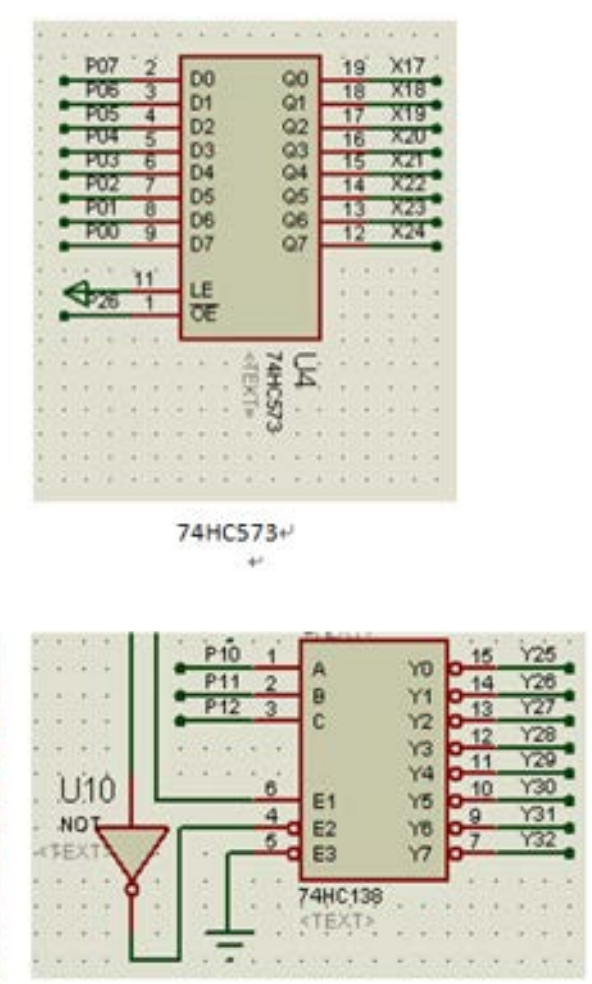

$74 \mathrm{HC} 138$

Fig. 2 System schematic

Light Pen and Detection Circuit. Infrared phototransistor homemade is used to make light pen, light pen detection circuit shown in Figure 4. Q 2 is a figure with an infrared phototransistor, used to complete the detection of the $32 \times 32$ dot matrix red LED lights on or off; R_6, R_p1 for Q_2 be limiting, the other can also be adjusted to increase or decrease R_p1 value of the output voltage; 
R_7, R_8 is used to U3A (comparator) phase ends with a reference voltage value, with the collection of information through its output voltage value

Compare (U_2> U_3, U_1 = U_min), R_12 U3A is output on the pull-up resistor. It works as follows: when the popular light to infrared phototransistor, infrared phototransistor resistance becomes smaller, its emitter voltage increases, then the 2-pin voltage is higher than 3 feet voltage, the comparator output is low 1 side. After charging the capacitor C_11 period of time, the comparator 2 feet to 3 feet voltage low voltage comparator 1 output is high end. Thus, when the optical signal is detected, the circuit generates a pulse signal. Due to the addition of the coupling capacitance in the circuit, it can effectively prevent the impact of environmental light pen. In this system, light pen is a very important aspect, in order to better stabilize the work, you must add the appropriate anti-jamming measures. In terms of signal transmission using shielded coaxial soft copper wire, infrared phototransistor peripheral jacket with black shrink tube, infrared optical transistor is smaller than the diameter of a single LED lights to ensure less interference from outside infrared.

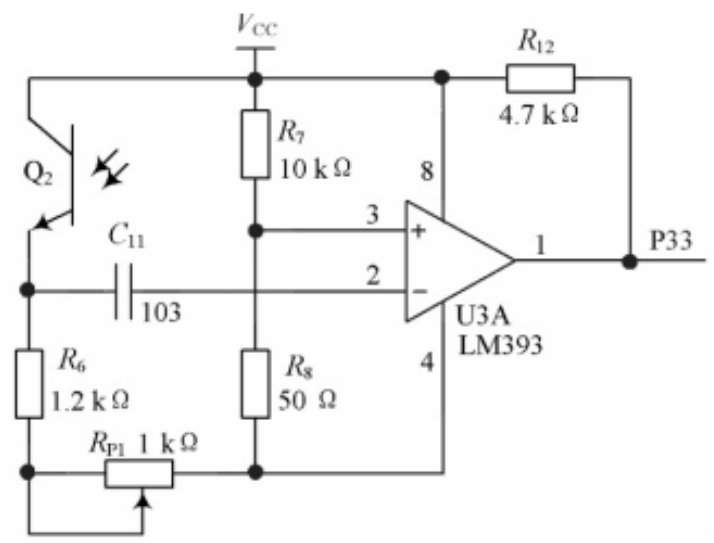

Fig. 3 Light pen detection circuit

LED Color Dot Matrix Display and Driver Circuit. LED color (red, green) lattice models are many, one of the most commonly used $4 \times 4,8 \times 8,16 \times 16$ modules, this design requires a $32 \times$ 32-color display, the display brightness, the volume of the designers define their own. Taking into account the factor purchases, choose $32 \times 32$ dot matrix display screen by the $168 \times 8$-color dot matrix module, use the 74HC154 and 74HC595 chips to drive the display, working from the serial port in Mode 0 (speed) will be displayed information quickly into the 74HC595.

LED is a non-linear component, both when it reaches a certain voltage value, by its own current will rise sharp. Consider $32 \times 32$ dot-matrix light-emitting diodes by the 1024 , when the current is large when they are lit simultaneously, it must consider the power supply capacity. General LED current value of $5 \sim 20 \mathrm{~mA}$, taking the maximum 20mA, simultaneously lit two lines, there are: $\mathrm{I}=$ $20 \times 64=1280 \mathrm{~mA}$.

Ambient Light Detection Circuit. Ambient light detecting circuit is shown in Figure 4. R_p photoresistor circuit in series with a fixed resistor R_9, the illumination change is converted to voltage changes, this voltage signal to analog to digital converter (A / D) by chip AD833, by the 5 feet into the microcontroller processing to control the brightness adjustment.

According to the partial pressure of principle, we are:

$$
\mathrm{V}_{\text {in }}=\frac{R_{9}}{R_{p}+R_{9}} V_{c c}
$$

Visible choose a different resistance $\mathrm{R} \_9$ can change the size of $\mathrm{V} \_$in of the input signal within the appropriate range. $\mathrm{R} \_\mathrm{p}$ photoresistor resistance change with changes in illumination is $3 \sim 4 \mathrm{k} \Omega$, by the above formula to calculate the $R_{-} 9$ is $3 \sim 4 \mathrm{~K} \Omega$, take $\mathrm{R}_{-} 9=4 \mathrm{~K} \Omega$. 


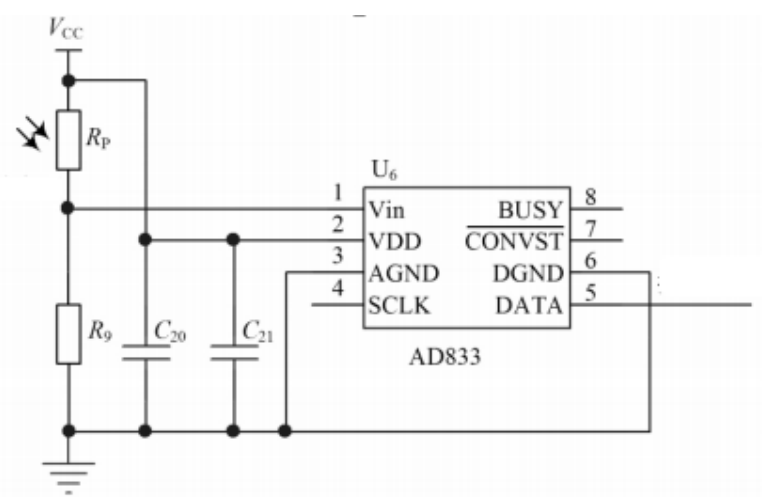

Fig. 4 External light detection circuit

Software Design. System software uses C language programming, software development and debugging in Keil uV3 programming environment. In order to facilitate writing and debugging, the program uses a modular design, based on the keys, light pen scanner, LED dot matrix display, LCD LCD, brightness adjustment and other functions are the design process, greatly simplifying the process design and commissioning work, save design cycle. The main program started internal function registers, ports, LCD initialization module, and then scanning the pen position in the main loop, LED display buffer data update, LED dot matrix output, the LCD module outputs, the visible brightness detection, low power consumption judgment and other functions. In the timer interrupt routine is carried out keypad scanning, complete the identification display mode, the corresponding information display mode and cursor coordinates are displayed on the LCD module.

\section{System Test}

The design of the test requirements and the results are shown in Table 1. By test results show that the system can achieve the lighting of the design requirements, zoned light, reverse display, the screen is cleared, strokes drag, rotate display, display brightness adjustment, sleep mode and other major functions.

Table 1 Test results

\begin{tabular}{|c|l|}
\hline $\begin{array}{c}\text { Test items } \\
\begin{array}{l}\text { Lit, of light, reverse display, the } \\
\text { screen is clear, }\end{array}\end{array}$ & \multicolumn{1}{|c|}{ Test Results } \\
\hline \multicolumn{1}{|c|}{ Object Drag } & $\begin{array}{l}\text { Complete lighting, zoned light, reverse display, the entire screen } \\
\text { clears. }\end{array}$ \\
\hline Ligatures multi-word & $\begin{array}{l}\text { You drag. First light pen to draw on the screen in bright fashion } \\
\text { delineation want dragging display objects, and then drag the stylus to } \\
\text { move the image to another location on the screen. }\end{array}$ \\
\hline $\begin{array}{l}\text { Optional erase mode can be combined in the 30s internal screen with } \\
\text { "scratched" wrote four Chinese characters, one by one (total not more } \\
\text { than 30 strokes), and stored in the machine, and then will be stored in } \\
\text { four words written on the screen by-turn display. }\end{array}$ \\
\hline $\begin{array}{l}\text { When the ambient light intensity } \\
\text { changes, can automatically and } \\
\text { continuously adjust the brightness of } \\
\text { the display on the screen. }\end{array}$ & $\begin{array}{l}\text { When the ambient light intensity changes, can automatically and } \\
\text { continuously adjust the brightness of the display on the screen. }\end{array}$ \\
\hline Timeout off display power saving & $\begin{array}{l}1 \sim 5 \text { min (This time is set by the controller) can automatically turn off } \\
\text { the display on the screen, and the entire system into hibernation, then } \\
\text { the system working current less than 5mA. }\end{array}$ \\
\hline
\end{tabular}

\section{Conclusion}

LED display design hardware consists of AT89C52 minimum system, $32 \times 32$ dot matrix display arrays, light pen and detection circuit, ambient light intensity detection circuit, a key input circuit, a liquid crystal display and other components. Dot red led is always working in dimly lit scan status, AT89C52 microcontroller use lit homemade light pen infrared phototransistor to detect light strokes and position the red led lights, calculated from the ranks of coordinate light pen position, and 
according to the key set led display control different operating modes, enabling lit, scratched, reverse display, clear the screen and other functions. Display according to ambient light intensity automatically adjusts the display brightness of the display, within a set time when the light pen is not touching the display or button is not pressed close all display, and the system enters hibernation, reduce power consumption, when a key Press when the system resumes. System software uses C language programming, software development and debugging in Keiluv3 programming environment. In order to facilitate writing and debugging, the program uses a modular design, based on the keys, light pen scanner, led dot matrix display, LCD, brightness adjustment and other functions are the design process, greatly simplifying the process design and commissioning work, save design cycle. Department of the main program started internal function registers, ports, LCD initialization module, and then scanning the pen position in the main loop, led display buffer data updating, led dot matrix output, the LCD module outputs, the visible brightness detection, low power consumption Analyzing and other functions. In the timer interrupt routine is carried out keypad scanning and complete the identification display mode, the corresponding information display mode and cursor coordinates are displayed on the LCD module.

In the design process of the system, using a combination of software and hardware, full software programming flexibility, and maximize mining microcontroller hardware resources. The system is cost-effective, stable operation, low energy consumption, showing good results.

\section{References}

[1] J.X. Gao. National Undergraduate Electronic Design Contest Training Tutorial Series. Beijing: Electronic Industry Press, 2009.

[2] Z.W. Huang. National Undergraduate Electronic Design Contest Training Course. Beijing: Electronic Industry Press, 2005.

[3] X.C. He. 365 Cases of Common Electronic Applications. Beijing: Electronic Industry Press, 2006.

[4] X. Zhang, C.P. Wang. C Language Program Design Basis. Beijing: Beijing Institute of Technology Press, 2006.

[5] R.G. Xu, Z.Z. Liao. Single-Chip Microcomputer Application Technology. Beijing: Mechanical Industry Press, 2007. 